\title{
A left hemisphere, but not right hemispace, advantage for tactual simultaneity judgments
}

\author{
MICHAEL E. R. NICHOLLS and ANNUKKA K. LINDELL \\ University of Melbourne, Parkville, Victoria, Australia
}

\begin{abstract}
Hemispheric asymmetries for tactile simultaneity judgments were investigated in 34 dextral adults. Pairs of vibrotactile stimuli with simultaneous or successive onsets were delivered unilaterally to the left or right hand. Participants made a forced-choice, bipedal response, indicating whether a stimulus was simultaneous or successive. The effect of hemispatial attentional biases was investigated, using ipsilateral (arms uncrossed) and contralateral (arms crossed) hand placements. Trials presented to the right hand were associated with fewer errors and a trend for faster response times than were those presented to the left hand. There was no asymmetry in response bias. Manipulations of hemispace did not affect the right hand advantage. These results confirm the existence of a left hemisphere temporal-processing advantage but fail to demonstrate that the asymmetry is the result of a rightward attentional bias. The implications of these results for absolute and relative models of hemispheric specialization are discussed.
\end{abstract}

Efron (1963a) conducted some of the first work specifically testing the proposition of a left hemisphere (LH) temporal-processing advantage. Efron required participants to make simultaneity judgments for pairs of stimuli presented in either the visual or the tactile modality. The stimuli were presented bilaterally, either to the left and right hands or to the left and right visual fields. The results revealed that simultaneity thresholds were lower when the right stimulus preceded the left than for the opposite order of presentation. Similar right-first advantages for bilateral visual stimuli have been reported by Corballis (1996) for simultaneity judgments and by St. John (1998) for temporal-order judgments. Within the auditory modality, Mills and Rollman (1980) required participants to make temporal order judgments for a pair of clicks delivered bilaterally to the left and right ears. Calculation of thresholds, using a method of constant stimuli, revealed that the left stimulus needed to precede the right by $4.4 \mathrm{msec}$ in order for simultaneity to be perceived.

Efron (1963a) and Mills and Rollman (1980) accounted for the right-first advantage with an absolute model of temporal-processing asymmetry. This model assumes that simultaneity and/or temporal order judgments are carried out exclusively in the LH. Thus, when stimuli are directed to the right side, they are transmitted directly, via the contralateral connections, to the temporal processor in the LH. Stimuli presented to the left side take a similar time to be transmitted to the right hemisphere (RH). However, there is an additional amount of time $(t)$ required for the message to cross the corpus callosum to reach the LH. Consequently, the time taken for stimuli presented to the left side to reach the temporal analyzer in the $\mathrm{LH}$ will be

Correspondence concerning this article should be addressed to M.E.R. Nicholls, Department of Psychology, University of Melboume, Parkville, VIC 3052, Australia (e-mail:m.nicholls@psych.unimelb.edu.au). $t$ msec longer than that for stimuli presented to the right side. For the case in which the right stimulus precedes the left stimulus by time $T$, the difference in arrival times at the $\mathrm{LH}$ would be equal to $T+t$. In contrast, when the left stimulus precedes the right by time $T$, the difference in arrival times would be equal to $T-t$. It can be seen that the temporal information available when the left stimulus precedes the right will be reduced as a function of the time required for the interhemispheric transmission of that information.

A major problem with the absolute specialization model, as proposed by Efron (1963a) and Mills and Rollman (1980), is that it predicts that no temporal-processing asymmetry should arise for unilateral stimulus presentations. This is because unilateral presentations can be processed without transcallosal transfer and, thus, are not distorted by transmission asynchronies. Despite this, there is ample evidence of an LH temporal-processing advantage for unilateral stimuli. For example, Nicholls (1994a) required participants to make a forced two-choice discrimination between two $130-\mathrm{msec}$ pulses of light, one of which contained a gap lasting between 8 and $14 \mathrm{msec}$. The stimuli were presented at random to either the left or the right visual field. The results revealed lower levels of error for the right visual field (and, hence, the LH) than for the left visual field. Participants responded gap more frequently and more quickly for trials presented to the right visual field than for those presented to the left visual field. These results combine to suggest that the $\mathrm{LH}$ was more able to resolve the temporal structure of the stimuli than was the RH. A similar LH advantage for gap detection has been reported for the auditory (Brown \& Nicholls, 1997; Nicholls, Schier, Stough, \& Box, 1999; Vroon, Timmers, \& Tempelaars, 1977) and tactile (Nicholls \& Whelan, 1998) modalities. An LH temporal-processing advantage for unilateral stimuli has been reported for a number of other tasks, including inspection time (Elias, Bulman- 
Fleming, \& McManus, 1999; Nicholls \& Cooper, 1991), simultaneity judgments (Nicholls, 1994a; St. John, 1998), and the perception of sequences (Hammond, 1981; Papcun, Krashen, Terbeek, Remmington, \& Harshman, 1974).

The LH advantage observed for unilateral stimulus presentations demonstrates that transmission asynchronies are not necessary for perceptual asymmetries to arise. This finding, however, does not rule out the possibility that $\mathrm{LH}$ specialization for temporal processing is absolute. Indeed, it is possible that stimuli that require temporal processing are always passed from the $\mathrm{RH}$ to the $\mathrm{LH}$. The process of transferring information from the $\mathrm{RH}$ to the LH may prolong the processing and degrade the quality of the stimuli, resulting in slower reaction times (RTs) and higher levels of error for stimuli presented to the RH than for stimuli presented to the LH (Kimura, 1966). However, there is a growing body of evidence that suggests that functional specialization between the hemispheres is relative, rather than absolute (Allen, 1983; Friedman \& Campbell-Polson, 1981). Thus, both hemispheres might be able to process temporal information, but the processing mechanisms of the $\mathrm{LH}$ would be more efficient than those of the RH. The perceptual asymmetries that arise with relative specialization are thought to reflect differences in the relative capacities of the hemispheres. Thus, for the case of temporal processing, stimuli presented to the right hand/ear/visual field are associated with low error rates and fast RTs because they are processed by the efficient mechanisms located in the LH. In contrast, stimuli presented to the left hand/ear/visual field are associated with higher error rates and longer RTs because they are processed by the inefficient processing centers located in the RH.

A number of neurostructural models have been proposed to account for a relative temporal-processing asymmetry. Miller (1996) has suggested that the LH contains more slow conducting, unmyelienated axons (total axonal conduction times $>100 \mathrm{msec}$ ) than does the RH and that the delays in transmission associated with these axons prevent the temporal structure of a stimulus from being integrated. Thus, slow neural conduction within the LH allows it to represent rapid temporal events. The RH, on the other hand, contains more fast-conducting myelinated axons (total axonal conduction times $<10 \mathrm{msec}$ ), which integrate temporal information and facilitate the representation of complex spatial information. In contrast to Miller's model, Nicholls (1996) has attributed the temporal-processing asymmetry to the intraregional and interregional neural organizations of the left and the right hemispheres, respectively (see Goldberg \& Costa, 1981, and Gur et al., 1980, for evidence of such an asymmetry). Nicholls (1996) proposed that the interregional organization of the RH increased the level of neural feedback and feedforward and that this blurred the temporal signal. In contrast, the intraregional organization of the $\mathrm{LH}$ was proposed to reduce the blurring of the signal, resulting in a more accurate representation of the temporal properties of a stimulus.
Despite the evidence that supports a temporal-processing asymmetry and the plausible neural models that can account for such an asymmetry, a number of researchers have questioned the existence of an LHtemporal-processing advantage (Efron, Yund, Nichols, \& Crandall, 1985; Grondin, 1998; Nagarajan, Blake, Wright, Byle, \& Merzenich, 1998; Sadler \& Deary, 1996). Most notable among this research is a series of studies carried out by Geffen and her colleagues (Clark \& Geffen, 1990; Geffen, Mason, Butterworth, McLean, \& Clark, 1996; Mason \& Geffen, 1996). In a series of carefully designed experiments, Geffen required participants to make a forced two-choice discrimination (simultaneous/successive) for pairs of punctate tactual stimuli lasting $9.5 \mathrm{msec}$. The stimulus onset asynchrony (SOA) required in order to discriminate between simultaneous and successive trials was calculated, using an adaptive staircase technique (parameter estimation by sequential testing, or PEST), which manipulated the difficulty of trials in line with the participants' previous response (Taylor \& Creelman, 1967). Despite manipulations that involved presenting the stimuli unilaterally and bilaterally (Clark \& Geffen, 1990; Geffen et al., 1996; Mason \& Geffen, 1996) and varying the predictability of the location of the stimulus (Mason \& Geffen, 1996), no evidence of a right hand (and, hence, $\mathrm{LH}$ ) advantage was found. The only condition in which a right hand advantage was found was for bilateral simultaneity judgments in a lateral hand placement condition (Geffen et al., 1996). Because this asymmetry was not evident in a midline hand placement condition, Geffen et al. suggested that the right hand advantage was due to a right hemispace advantage, rather than to an $\mathrm{LH}$ processing advantage. A general tendency to attend more to the right hemispace is supported by a number of researchers (Bradshaw, Bradshaw, PiersonSavage, \& Nettleton, 1988; Reuter-Lorenz, Kinsbourne, \& Moscovitch, 1990).

Geffen et al.'s (1996) proposition that perceptual asymmetries for temporal processing are the result of a general rightward attentional bias, rather than of a $\mathrm{LH}$ temporal-processing advantage, has important implications for models of temporal-processing asymmetry. Bearing this in mind, the present study sought to replicate the effect of hemispace observed by Geffen et al. for tactile simultaneity judgments. The task used in the present study was based on a visual simultaneity study conducted by Nicholls (1994a) that yielded a right visual field advantage. In the present study, pairs of vibrotactile stimuli were delivered at random to either the left or the right hand. For half of the trials, the onset of the stimuli was simultaneous. For the other half of trials, the onset of the stimuli was separated by an SOA lasting 24, 36, 48 , or $60 \mathrm{msec}$. The different SOAs were varied between blocks. The participants were informed that $50 \%$ of the trials were successive and were asked to make a forced two-choice discrimination (simultaneous/successive) for each trial. In line with the results of Nicholls (1994a), it was expected that (1) error rates would be lower for the right hand than for the left hand, (2) RTs would be faster for the right hand 
than for the left hand for the successive stimuli, and (3) response bias toward simultaneous responses would be higher for the left hand than for the right hand. These asymmetries would presumably reflect the more efficient temporal processing of stimuli presented to the right hand than of those presented to the left hand. The effect of hemispace was investigated with ipsilateral (arms uncrossed) and contralateral (arms crossed) hand placement conditions. If perceptual asymmetries for simultaneity judgments are the result of a rightward attentional bias, there should be a right hand advantage for the ipsilateral condition and a left hand (or a reduced right hand) advantage for the contralateral condition. If the perceptual asymmetry is the result of a $\mathrm{LH}$ processing advantage, the right hand advantage should persist despite manipulations of hemispace.

There are two reasons why the simultaneity judgment task used in the present study might be expected to generate an LH advantage, whereas the task used by Geffen et al. (1996) did not. First, the present study provided participants with a consistent definition of what constituted successive and simultaneous events within a block of testing. It was hoped that this stability within blocks would encourage the participants to adopt a stable decision criterion, which in turn, would reduce the levels of noise associated with the discrimination. The low level of noise associated with the task should allow (perhaps, small) hemispheric asymmetries to emerge in the data. The staircase method used by Geffen et al. varied the nature of the successive stimuli (or signals) between trials. This variability may have discouraged the use of a stable decision criterion. As a result, the higher levels of noise associated with responses may have obscured any hemispheric asymmetry.

The second potentially important difference between the present study and the studies conducted by Geffen is related to the duration of the stimuli. It is possible that the symmetry observed by Geffen et al. (1996) was related to task-related features of their experiments that facilitated the perception of apparent movement. Fraisse (1978) reported that the perception of apparent (or phi) movement occurs more readily for stimuli lasting less than $100 \mathrm{msec}$. For tactile vibratory stimuli lasting $10 \mathrm{msec}$, Kirman (1974) found that optimal apparent movement occurred for interstimulus onset intervals of $50 \mathrm{msec}$. This interstimulus interval falls within the range $(29-55 \mathrm{msec})$ of threshold SOAs reported by Geffen et al. As a result, the perception of apparent movement may have played an important role in Geffen's task. Evidence of hemispheric specialization for the detection of apparent movement is unclear. Jasper and Raney (1937) and Carter (1953) reported an LH advantage, whereas Bertoloni, Anzola, Buchtel, and Rizzolatti (1978) and Eals (1987) found an RH advantage for the processing of movement. The lack of a consistent LH advantage for the detection of apparent movement may be related to the way in which the stimuli are processed. Gregory and Harris (1984) reported that visual apparent motion was represented in the nervous system in a spatial code, similar to that observed for real movement. The spatial code produced through the perception of apparent movement would not be expected to favor one hemisphere over the other. Thus, spatial coding of the stimuli, rather than a lack of temporal-processing asymmetry, may have caused the symmetry reported by Geffen et al. (1996).

The present study sought to limit the contribution of apparent-motion cues by using vibrotactile stimuli lasting more than $480 \mathrm{msec}$. The duration of these stimuli fall well beyond the 100-msec mark that Fraisse (1978) suggested was the maximum normally required for the perception of apparent movement. In addition, Kirman (1974) has reported that increases of stimulus duration cause an increase in the optimal interstimulus interval required for the perception of tactile apparent movement. For stimuli lasting $200 \mathrm{msec}$, the optimal interstimulus interval for the perception of apparent movement was $125 \mathrm{msec}$. The vibrotactile stimuli used in the present study (which lasted $480 \mathrm{msec}$ or more) would be expected to yield an optimal interstimulus interval in excess of $125 \mathrm{msec}$. This interval is well above the range of SOAs that would be expected to be critical to the simultaneity judgment task used in the present study.

\section{METHOD}

\section{Participants}

Thirty-four (13 males, 21 females) first-year psychology students participated in this study as part of their course requirements. All the participants were naive as to the aims and expected outcomes of the study. The modal age was 18 years, and all the participants were right-handed, as measured by the Edinburgh Handedness Inventory (Oldfield, 1971).

\section{Apparatus}

The experiment was controlled with an IBM clone interfaced with a digital input/output card with an on-board 1.0-msec timer (Blue Chip Technology, DCM-16). Four tactile stimulators were constructed from $100-\mathrm{mm}, 5-\mathrm{W}$ oval speakers. The paper surrounding the center of the speaker was removed to free the movement of the central coil. A plastic plunger was adhered to the central diaphragm and was used to transfer movement from the speaker to the palmar surface of the finger. The upper point of the plunger was machined to a point of $0.9 \mathrm{~mm}$ at an angle of $35^{\circ}$. The tip of the plunger passed though an $8-\mathrm{mm}$ hole in a sheet of perspex located over the top of the speaker body. The participants' responses were recorded by using four foot-pedals (Radio Spares, No. 316-901). A pedal was located under the heel and toe of each foot. Lifting either the toes or the heels of both feet activated the switches. Velcro attachments allowed the pedals to be adjusted for different-sized feet. White noise was delivered at an intensity of $74 \mathrm{~dB}$ SPL to the left and right ears through a set of headphones (Sennheiser HD40) in order to mask noises from the tactile stimulators.

\section{Stimuli}

In an unactivated state, the tip of the central plunger was level with the outer casing. When the speaker was activated, the plunger was driven $1 \mathrm{~mm}$ past the outer casing. The tactile stimulators were driven with square wave pulses at a frequency of $166.67 \mathrm{~Hz}$. This frequency produced tactile stimulation that was clearly perceptible but not uncomfortable. For simultaneous trials, the stimuli were de- 
livered simultaneously to the index and middle fingers of either the left or the right hand. Both stimulators were deactivated once $540 \mathrm{msec}$ had passed. In the successive condition, the onsets of the stimuli were separated by an SOA lasting $24,36,48$, or $60 \mathrm{msec}$. The stimulators were simultaneously deactivated once $540 \mathrm{msec}$ has passed since the time of initial stimulation. RTs were measured from the onset of the first signal in all of the conditions.

By making the offset of the stimuli simultaneous in the successive condition, the duration of each stimulus within the pair was slightly different. Thus, for successive trials with an SOA of $60 \mathrm{msec}$, the duration of the first stimulus was $540 \mathrm{msec}$, whereas the duration of the second stimulus was $(540-60) 480 \mathrm{msec}$. The advantage of this technique is that it limits the temporal information contained in the stimulus to the onset of the stimulus. The disadvantage of the technique is that the $540-\mathrm{msec}$ stimulus may feel, overall, stronger than the 480 -msec stimulus. As a result, the participants may respond successive when they feel a difference in intensity between the stimuli. To investigate this possibility, a pretest was conducted that required the participants to discriminate between the intensities of trials that were either 540 or $480 \mathrm{msec}$ long. Seven participants were required to complete 48 trials, $50 \%$ of which were either short or long. An analysis of the results revealed an error rate of $41.3 \%$, which was significantly better than chance $[t(6)=3.33, p<.05]$. However, the error rate was significantly higher than the rate observed when the stimulus could be differentiated on the basis of a $60-\mathrm{msec}$ SOA (error $=19.6 \%$ ). Thus, it would appear that participants can use intensity cues to distinguish between stimuli. However, the level of performance associated with these cues is much lower than when SOA is used to differentiate the stimuli.

\section{Procedure}

Each trial began with the illumination of a light-emitting diode placed $500 \mathrm{~mm}$ in front of the participant's midline. After a warning period lasting between 900 and $1,100 \mathrm{msec}$, a pair of tactile stimuli was delivered unilaterally to the middle and index fingers of either the left or the right hand. The participant made a bipedal response by raising either the toes or the heels of both feet. Half of the participants responded simultaneous by lifting their toes and successive by lifting their heels. The order of response was reversed for the remaining participants. A trial in which the participant took longer than $1,500 \mathrm{msec}$ to respond was automatically rejected by the computer program and replaced with an identical trial inserted at random into the block. If the participants consistently took too long to make their responses, they were encouraged to respond more quickly. In general, the participants had fewer than eight trials per session rejected because of long RTs. A new trial was commenced $1,500 \mathrm{msec}$ following the response. White noise was presented binaurally throughout the testing session.

A total of 480 trials was administered to each participant in eight blocks of 60 trials. Four consecutive blocks were administered with participants' hands located $45^{\circ}$ to the left or right of their midline in the ipsilateral hemispace. The other four blocks were administered with the participants' arms crossed so that their hands were located $45^{\circ}$ in the contralateral hemispace. The forearm that lay over the other in the contralateral condition was varied between participants. The tactile stimulators were placed together in pairs and were positioned so that the index and middle fingers of one hand could reach them. Each pair of stimulators was turned $90^{\circ}$ between the ipsilateral and the contralateral conditions, to accommodate the different orientations of the hands. The stimulators were rotated clockwise after each participant to eliminate the effect of possible mechanical differences between the stimulators. The four levels of SOA $(24,36,48$, and $60 \mathrm{msec})$ were varied between blocks and were repeated for each hand placement condition. The order in which the different hand placements and SOAs were administered was balanced between participants, using a pseudo-Latin-squares design. Within each block, equal numbers of trials were delivered to the left and the right hands, and equal numbers of simultaneous and successive stimuli were delivered. For half of the participants, the successive trials consisted of the index finger's being stimulated before the middle finger. The order of finger stimulation was reversed for the remaining participants. The order in which the fingers were stimulated was balanced between participants, to counteract any advantage in reporting left-to-right, as opposed to right-to-left, movement. The participants were advised whether they had been allocated to the middle-first or the index-first condition. This method of presentation ensured that the choice was always limited to two possibilities. The order in which the within-block factors of side and stimulus type occurred was randomized for each participant.

Each participant attended one 50-min session of testing, during which the handedness questionnaire and the experimental task were completed. The experiment was conducted in a well-lit, quiet room. The participants were informed that the trials would take one of two forms: one in which the onset of the vibrations was simultaneous, or one in which the onset of the vibrations was successive. They were informed that there would be equal numbers of both types of trials. The participants were told that the primary requirement of the experiment was to be accurate but were also encouraged to respond as quickly as they could.

Prior to the experimental trials, the participants were given a number of practice trials in order to familiarize them with the equipment and the experimental procedure. Four blocks of 16 practice trials were presented. The practice blocks began at an easy level and became progressively more difficult, with SOAs of 240,120 , 96 , and $60 \mathrm{msec}$, respectively. The experimental trials commenced after each participant had reached a level of accuracy that demonstrated that they could cope with the demands of the task ( $80 \%$ correct responses at an SOA of $60 \mathrm{msec}$ ).

\section{RESULTS}

\section{Response Error}

A measure of response error was calculated by adding the number of errors for the simultaneous stimuli to the number of errors for the successive stimuli. Error scores were converted to a percentage by dividing the scores by the total number of trials within each condition and multiplying by 100 . The error data were analyzed with a repeated measures analysis of variance (ANOVA), with SOA $(24,36,48$, and $60 \mathrm{msec}$ ), hand (left, right), and position (ipsilateral, contralateral) as within subjects factors.

The error data are represented in Figure 1. Error rates decreased as a function of increases in SOA, and this produced a significant main effect of SOA $[F(3,99)=62.44$, $p<.001]$. Error rates were lower for the right hand than for the left hand $[F(1,33)=4.22, p<.05]$. Error rate did not change as a function of hand position $[F(1,33)=1.48$, n.s.]. The interaction between hand and position was not significant $[F(1,33)=0.15$, n.s. $]$, nor were there any other interactions.

\section{Response Bias}

A simple measure of response bias was calculated by subtracting the number of successive responses from the number of simultaneous responses, irrespective of whether or not they were correct. A negative score indicates a bias toward successive responses, whereas a positive score indicates a bias toward simultaneous responses. Scores could range from a minimum of -30 to a maximum of 


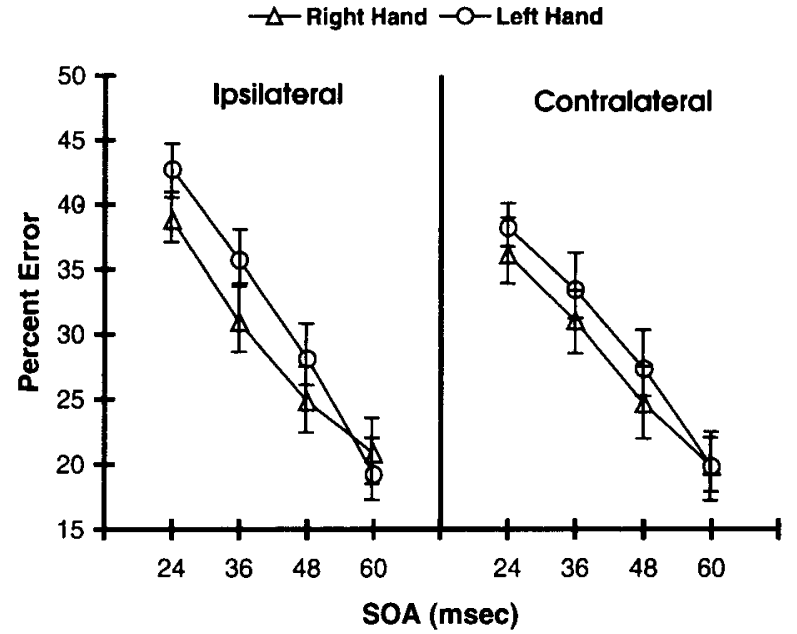

Figure 1. Mean percentages of error (with $\pm S E$ bars) for the right and left hands across the different stimulus onset asynchronies (SOAs) and hand placement conditions.

+30 . The response bias data were analyzed, using the procedure described for the error data.

The response bias data are represented in Figure 2. Response bias decreased as a function of increases in SOA $[F(3,99)=24.70, p<.001]$. An inspection of Figure 2 suggests that the bias toward simultaneous responses may have been stronger for the left hand than for the right hand. However, the main effect of hand was not significant $[F(1,33)=1.26$, n.s. $]$. The response bias toward simultaneous responses was higher in the ipsilateral than in the contralateral condition $[F(1,33)=9.06, p<$ $.01]$. Figure 2 suggests that the response bias may have been higher for the left hand than for the right hand for the ipsilateral, but not for the contralateral condition. However, the interaction between position and hand was not significant $[F(1,33)=0.10$, n.s. $]$. The lack of interaction was confirmed by post hoc analyses that revealed no difference between the hands for the ipsilateral $[F(1,33)=$ 1.12$, n.s. $]$ or the contralateral $[F(1,33)=0.44$, n.s. $]$ condition. No other interaction approached statistical significance.

\section{Signal Detection Analysis}

The error and response bias measures provide simple measures of performance that have yielded stable asymmetries in the past (e.g., Brown \& Nicholls, 1997; Nicholls $\&$ Whelan, 1998). However, the error and response bias measures are confounded to some degree. Shifts from an ideal response bias of zero will increase the likelihood of errors. Thus, it is possible that the asymmetry observed for the error data could reflect differences in response bias between the hands, rather than an asymmetry in error rate per se. Independent measures of discriminability and response criterion were gained with a signal detection analysis, using the procedure described by $\mathrm{McNicol}$ (1972) for a yes-no task. Successive responses were interpreted as yes (signal) responses, whereas simultaneous responses were interpreted as no (noise) responses. To provide more stable data for the signal detection analysis, the data were collapsed across the contralateral and ipsilateral hand positions. The effect of extreme scores, in which $P(\mathrm{Ss})$ or $P(\mathrm{Sn})$ was equal to 0.0 or 1.0 , was avoided by rounding these scores to a $P$ value of .033 and .966 , respectively. The $d^{\prime}$ and $\beta$ values, together with their standard deviations, are shown in Table 1.

The $d^{\prime}$ data were analyzed with a repeated measures ANOVA, with side and SOA as within-subjects factors. Values of $d^{\prime}$ were significantly higher for the right hand than for the left hand $[F(1,33)=4.19, p<.05]$. There was also a significant effect of SOA where $d^{\prime}$ values increased as a function of increases in $\operatorname{SOA}[F(3,99)=58.46, p<$ $.001]$. There was no interaction between SOA and hand $[F(3,99)=1.54$, n.s. $]$. Analysis of the $\beta$ values revealed no significant main effect for $\operatorname{SOA}[F(3,99)=0.43$, n.s. $]$ or hand $[F(1,33)=0.31$, n.s. $]$ and no interaction between SOA and hand $[F(3,99)=2.61$, n.s. $]$.

\section{Reaction Time}

Mean RT for correct responses was calculated within each of the conditions. The effect of outliers was reduced by excluding (and then repeating) trials with RTs greater than $1,500 \mathrm{msec}$ during the experiment. Mauchly sphericity tests revealed that assumptions of homogeneity of variance were satisfied for all main effects. The data were analyzed with a repeated measures ANOVA, with SOA $(24,36,48$, and $60 \mathrm{msec}$ ), hand (left, right), position (ipsilateral, contralateral), and stimulus type (simultaneous, successive) as within-subjects factors.

The RT data for simultaneous and successive stimuli are shown in Figures 3A and 3B, respectively. RTs decreased as a function of increases in $\operatorname{SOA}[F(3,99)=7.77$, $p<.001]$. The trend for RTs to be faster for the right hand

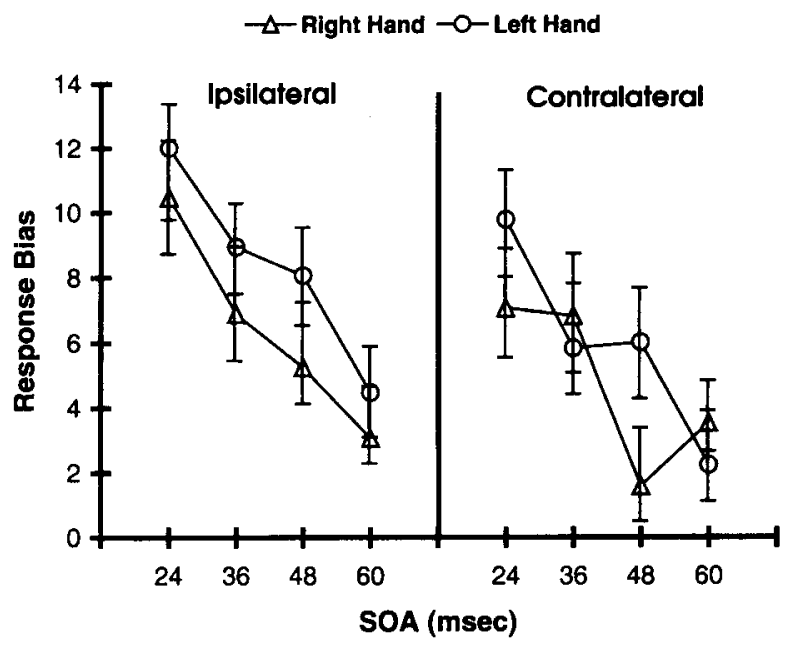

Figure 2. Mean response biases (with $\pm S E$ bars) for the right and left hands across the different stimulus onset asynchronies (SOAs) and hand placement conditions. Higher response bias scores reflect an increased bias toward simultaneous responses. 


\begin{tabular}{|c|c|c|c|c|c|c|c|c|c|}
\hline \multicolumn{10}{|c|}{$\begin{array}{l}\text { Table } 1 \\
\text { Mean } d^{\prime} \text { and } \beta \text { Values (With Standard Deviations) } \\
\text { for the Left and Right Hands Across the } \\
\text { Different Stimulus Onset Asynchronies }\end{array}$} \\
\hline \multirow[b]{3}{*}{ Measure } & \multirow[b]{3}{*}{ Hand } & \multicolumn{8}{|c|}{ Stimulus Onset Asynchrony } \\
\hline & & \multicolumn{2}{|c|}{$24 \mathrm{msec}$} & \multicolumn{2}{|c|}{$36 \mathrm{msec}$} & \multicolumn{2}{|c|}{$48 \mathrm{msec}$} & \multicolumn{2}{|c|}{$60 \mathrm{msec}$} \\
\hline & & $M$ & $S D$ & $M$ & $S D$ & $M$ & $S D$ & $M$ & $S D$ \\
\hline \multirow[t]{2}{*}{$d^{\prime}$} & Left & 0.61 & 0.51 & 0.96 & 0.81 & 1.45 & 0.81 & 1.99 & 0.88 \\
\hline & Right & 0.83 & 0.69 & 1.20 & 0.84 & 1.63 & 1.00 & 1.93 & 0.95 \\
\hline \multirow[t]{2}{*}{$\beta$} & Left & 1.63 & 0.98 & 1.73 & 1.22 & 2.20 & 1.62 & 1.90 & 1.51 \\
\hline & Right & 1.89 & 1.45 & 1.71 & 1.16 & 1.65 & 1.28 & 1.67 & 0.88 \\
\hline
\end{tabular}

than for the left hand approached significance $[F(1,33)=$ $3.10, p<.1]$. RT did not change as a function of position $[F(1,33)=0.02$, n.s. $]$. RTs to successive stimuli were slower than those to simultaneous stimuli $[F(1,33)=10.38$, $p<.005]$. An inspection of Figures $3 \mathrm{~A}$ and 3B suggests that responses were faster for the right hand than for the left hand for successive trials, but not for simultaneous trials. The interaction between hand and stimulus type was not significant $[F(1,33)=1.66$, n.s. $]$. Post hoc examinations are usually not justified when an interaction fails to reach statistical significance. However, given that Nicholls (1994a) found faster RTs for the LH for the detection of simultaneous trials, but not of successive trials, it was felt that post hoc analyses were justified in this case. Examination of the data for successive stimuli in isolation revealed faster RTs for the right hand than for the left hand $[F(1,33)=4.46, p<.05]$. There was no asymmetry between the hands for the simultaneous stimuli $[F(1,33)=$ 0.22 , n.s.]. A significant interaction arose between SOA and stimulus type $[F(3,99)=3.64, p<.05]$. An inspection of Figures $3 \mathrm{~A}$ and $3 \mathrm{~B}$ suggests that this interaction was the result of an RT advantage for simultaneous, as compared with successive, stimuli that decreased as a function of increases in SOA. Post hoc analyses confirmed this interpretation by demonstrating an effect of stimulus type for SOAs of $24[F(1,33)=13.92, p<.005]$ and 36 $[F(1,33)=7.24, p<.05] \mathrm{msec}$, but not for SOAs of 48 $[F(1,33)=1.65$, n.s. $]$ and $60[F(1,33)=1.97$, n.s. $]$ msec. The interaction between hand and position was not significant $[F(1,33)=0.09$, n.s. $]$, nor were there any other interactions.

\section{DISCUSSION}

The effect of SOA on the error, response bias, signal detection, and RT data was very similar to that reported by Nicholls (1994a) for visual simultaneity judgments. Decreases in SOA increased the difficulty of the task and resulted in higher levels of error, slower RTs, and reduced $d^{\prime}$ values. Decreases in SOA also increased the bias toward simultaneous responses. This change in response bias most likely reflects the fact that the successive stimuli came to resemble the simultaneous stimuli at the shorter SOAs. As a result, there was an increased probability that the participants would misclassify the successive stim- uli as simultaneous. Despite this change in response bias, estimates of $\beta$ demonstrated that the participants' response criterion was stable across the different SOAs.

Fewer errors were made for trials presented to the right hand than for those presented to the left hand. Analysis of the response bias data revealed no significant difference between the hands. However, the difference between the hands was in the predicted direction, with more simultaneous responses being made for the left hand than for the right hand. The nonsignificant result observed for the response bias data might reflect high levels of noise caused by idiosyncratic response strategies adopted by the participants. Signal detection measures revealed larger $d^{\prime}$ values for the right hand than for the left hand but no asymmetry for $\beta$. These results effectively rule out the possibility that the lower error levels for the right hand were the result of a reduced bias toward simultaneous responses. Rather, it would appear that the right hand is genuinely more sensitive in discriminating successive from simultaneous events. There was a tendency for RTs to be faster for trials presented to the right hand than for those presented to the left hand, which approached statistical significance. When the data from simultaneous and successive trials were analyzed separately, a significant right hand advantage arose for successive, but not for simultaneous trials. A similar $\mathrm{LH}$ advantage for detection of successive stimuli, but not of simultaneous stimuli, has been reported by Nicholls (1994a) for visual simultaneity judgments. It should be noted, however, that the interaction between stimulus type and hand was not significant in the present study. Thus, the right hand advantage for the detection of successive stimuli should be interpreted with some caution.

Taken together, the error, response bias, RT, and signal detection data support a right hand advantage for simultaneity judgments. The small effect observed for the RT data and the nonsignificant effect observed for the response bias data suggest that the right hand advantage is relatively weak. This weakness may explain the inconsistency of results reported for simultaneity judgments within the tactile modality (e.g., Geffen et al., 1996).

RTs to the simultaneous stimuli were faster than those to the successive stimuli at the shorter SOAs, but not at the longer SOAs. The lack of difference between the stimuli at the longer SOAs suggests that the simultaneous and successive stimuli were detected with equal ease. At the shorter SOAs, however, RTs to simultaneous stimuli were faster than those to successive stimuli. The RT advantage for simultaneous stimuli may be related to the high proportion of simultaneous responses made at the shorter SOAs. Because simultaneous responses were the default at the shorter SOAs, the participants may have been surer of these responses than of successive responses. A higher level of confidence for simultaneous responses is likely to have led to faster RTs to these stimuli.

Axelrod, Thompson, and Cohen (1968) reported that simultaneity thresholds increased progressively across midline, ipsilateral, and contralateral hand placement condi- 
A
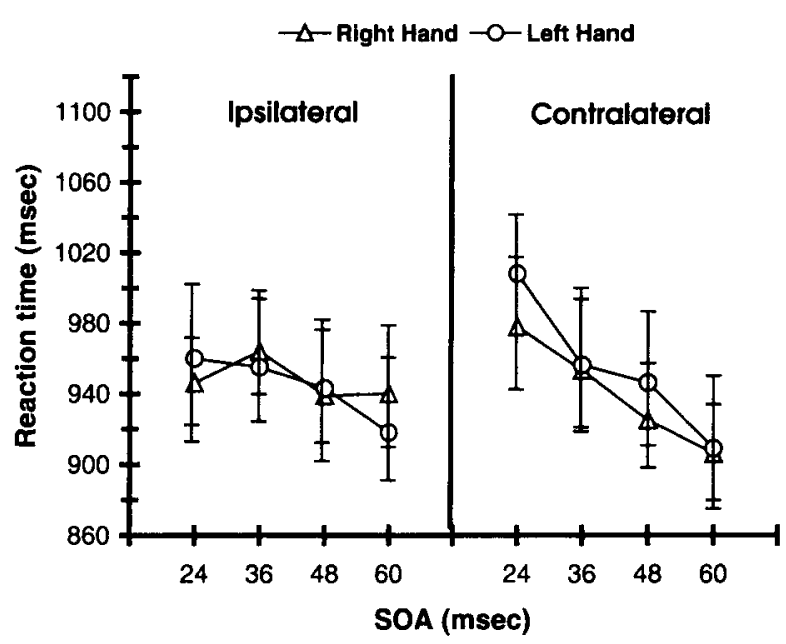

B

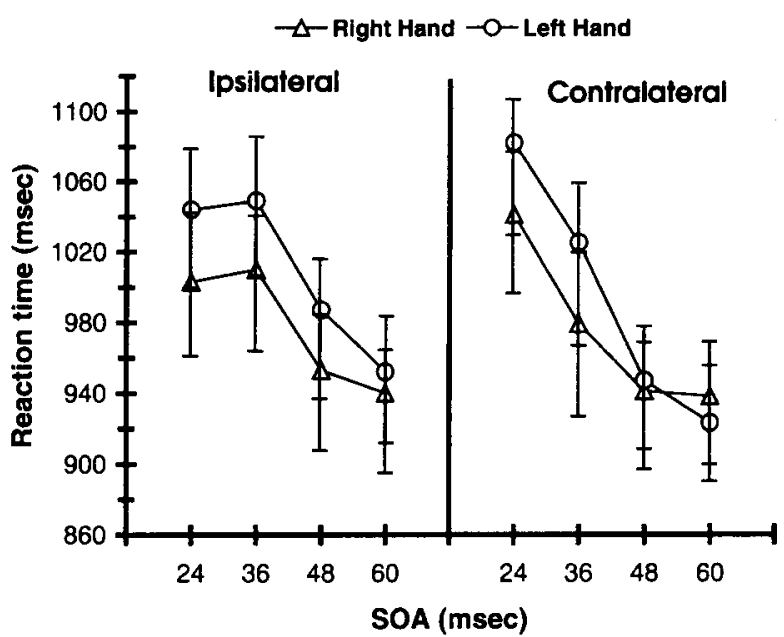

Figure 3. Mean reaction times (with $\pm S E$ bars) for correct responses to trials directed to the right or the left hand across the different SOAs for (A) simultaneous and (B) successive trials.

tions. The present study found no increase in RT or error for the contralateral condition, as compared with the ipsilateral condition. Similar results have been reported by Geffen et al. (1996) for simultaneity thresholds. An analysis of the response bias data, however, revealed that more simultaneous responses were made in the ipsilateral condition than in the contralateral condition. It is difficult to know what might have caused this change in response bias. It is unlikely that the difference is related to a coding conflict in response preparation (Nicoletti, Anzola, Luppino, Rizzolatti, \& Umiltà, 1982), because the response (lifting either both toes or both heels) was orthogonal to the stimulus (left or right). The difference also cannot be attributed to changes in which finger (middle vs. index) was stimulated first, because this factor was varied be- tween participants. One plausible explanation is that holding the arms in their ipsilateral space is more usual than crossing them, as in the contralateral condition. The novelty and extra attentional load associated with the contralateral condition may have encouraged the participants to adopt a different strategy that involved making fewer default simultaneous responses.

Manipulations of hemispace had no effect on the degree or the direction of the asymmetry for the error, response bias, or RT data. These results suggest that a general rightward attentional bias of the type described by Geffen et al. (1996) does not play an important role in the right hand advantage for tactual simultaneity judgments. Rather, the right hand advantage would appear to be the result of an LH temporal-processing advantage. It is possible that aspects of the experimental technique used by Geffen et al. prevented them from observing a consistent right hand advantage. The method of constant stimuli used in the present study may have provided a more stable basis for detecting hemispheric asymmetries. In addition, the use of stimuli with durations in excess of $100 \mathrm{msec}$ may have discouraged the use of apparent-motion cues (Fraisse, 1978).

The results of the present study corroborate research conducted by Nicholls (1994b) that investigated the effects of attentional biases on perceptual asymmetries for temporal processing. The study used central arrow cues to direct attention to the left or right hemispaces during a divided visual field gap-detection task. Measures of error, $\mathrm{RT}$, and response bias revealed a temporal-processing advantage for stimuli presented to the right visual field. In addition, there were significant costs and benefits associated with the invalid and the valid spatial cues (respectively). However, the cuing procedure had no effect on the degree or the direction of the asymmetry. If the right visual field advantage was the result of a rightward attentional bias, the asymmetry should have been reduced in the cued conditions, in which attention was balanced between the visual fields, as compared with the neutral condition. Nicholls' (1994b) research, together with the results of the present study, suggests that rightward attentional biases are not an important factor in visual and tactile temporal-processing asymmetries.

Clinical research also suggests that temporal-processing asymmetries are not the result of a rightward attentional bias. This research typically investigates hemispheric asymmetry by comparing performances between patients with unilateral lesions to the left or the right hemispheres. As a result, hemispatial attentional biases should play little or no role, since stimuli are usually presented along the participant's midline. For visual and auditory simultaneity judgments, Efron (1963b) found that LH aphasics required an SOA of 277 and $474 \mathrm{msec}$ (respectively) to reach a $100 \%$ correct threshold. These SOAs were significantly longer than the SOAs reported for nonaphasic/RH patients, with thresholds of 115 and $130 \mathrm{msec}$ for the visual and the auditory modalities, respectively. For a visual temporal order task, Swisher and Hirsh (1972) reported 
that fluent and nonfluent aphasics required 170 and $116 \mathrm{msec}$ (respectively) to determine the order of two visual stimuli presented at the midline. Patients with $\mathrm{RH}$ damage required a significantly shorter SOA $(66 \mathrm{msec})$ to make the same discrimination. A deficit following $\mathrm{LH}$ damage has been reported for a variety of other tasks, including two-click fusion (Lackner \& Teuber, 1973), the identification of sequences (Carmon \& Nachshon, 1971), flicker fusion (Goldman, Lodge, Hammer, Semmes, \& Mishkin, 1968), and the temporal integration of sequences (Szelag, Steinbüchel, \& Pöppel, 1997). Although the majority of the evidence does appear to support a temporalprocessing deficit following LH damage (Nicholls, 1996), it should be noted that this support is not unanimous (Aram \& Ekelman, 1988; Efron et al., 1985; Parsons, Majumder, \& Chandler, 1967).

Efron (1963a) and Mills and Rollman (1980) both suggested that perceptual asymmetries for temporal processing were the result of transmission asynchronies between the hemispheres. The present study avoided the effect of transmission asynchronies by presenting the stimuli unilaterally to the hemispheres. Despite this, an LH advantage for temporal processing emerged. This result suggests that timing asynchronies associated with the transcallosal passage of information are not central to perceptual asymmetries for temporal processing. The asymmetry in the present study could reflect absolute or relative $\mathrm{LH}$ specialization for temporal processing. If the specialization were absolute, the perceptual asymmetry would reflect the degradation of the stimulus as it passes from the RH to the LH. In contrast, if the specialization were relative, the perceptual asymmetry would reflect differences between the hemispheres in their capacity to process temporal information.

Although it is beyond the scope of the present study to test the relative and absolute specialization models, it should be noted that general research into human cerebral asymmetry suggests that hemispheric specialization is relative, rather than absolute (Allen, 1983; Friedman \& Campbell-Polson, 1981). In addition, research in which the effect of unilateral lesions on temporal-processing ability has been investigated demonstrates that the RH is capable of some (albeit, imprecise) temporal processing (Efron, 1963b; Lackner \& Teuber, 1973; Swisher \& Hirsh, 1972). The relative and absolute models could be tested more directly by comparing separate left hand and right hand responses for lateralized presentations (see, Moscovitch, 1986 , for an explanation of this method) or by investigating relative hemispheric activity with electroencephalographic techniques.

\section{REFERENCES}

Allen, M. (1983). Models of hemispheric specialization. Psychological Bulletin, 93, 73-104.

Aram, D. M., \& Ekelman, B. L. (1988). Auditory temporal perception of children with left or right brain lesions. Neuropsychologia, 26, 931-935. AXelrod, S., Thompson, I. W., \& Cohen, L. D. (1968). Effects of senescence on the temporal resolution of somesthetic stimuli presented to one hand or both. Journal of Gerontology, 23, 191-195.
Bertoloni, G., Anzola, G. P., Buchtel, H. A., \& Rizzolatti, G. (1978). Hemispheric differences in the discrimination of the velocity and duration of a simple visual stimulus. Neuropsychologia, 16, 213220.

Bradshaw, J. L., Bradshaw, J. A., Pierson-Savage, J. M., \& NetTLETON, N. C. (1988). Overt and covert attention and vibrotactile reaction times: Gaze direction, spatial compatibility and hemispatial asymmetry. Canadian Journal of Psychology, 42, 44-56.

Brown, S., \& Nicholls, M. E. R. (1997). Hemispheric asymmetries for the temporal resolution of brief auditory stimuli. Perception \& Psychophysics, 59, 442-447.

CARMON, A., \& NACHSHON, I. (1971). Effect of unilateral brain damage on perception of temporal order. Cortex, 7, 410-418.

CARTER, D. B. (1953). A further demonstration of phi movement cerebral dominance. Journal of Psychology, 36, 299-309.

Clakk, C. R., \& GefFen, G. M. (1990). Hemispheric equivalence for simultaneity judgments of somatosensory stimuli. Cognitive Neuropsychology, 7, 311-327.

Corballis, M. C. (1996). Hemispheric interactions in temporal judgments about spatially separated stimuli. Neuropsychology, 10, 42-50.

EALS, M. (1987). Asymmetric processing in perception of apparent movement. Neuropsychologia, 25, 429-434.

EFron, R. (1963a). The effect of handedness on the perception of simultaneity and temporal order. Brain, 86, 261-283.

EFron, R. (1963b). Temporal perception, aphasia and déjà vu. Brain, 86, $403-424$

Efron, R., Yund, E. W., Nichols, D., \& Crandall, P. H. (1985). An ear asymmetry for gap detection following anterior temporal lobectomy. Neuropsychologia, 23, 43-50.

Elias, L. J., Bulman-Fleming, M. B., \& McManus, I. C. (1999). Visual temporal asymmetries are related to asymmetries in linguistic perception. Neuropsychologia, 37, 1243-1249.

Fraisse, P. (1978). Time and rhythm perception. In E. C. Carterette \& M. P. Friedman (Eds.), Handbook of perception (Vol. 3, pp. 203-254). New York: Academic Press.

Friedman, A., \& Campbell-Polson, M. (1981). Hemispheres as independent resource systems: Limited-capacity processing and cerebral specialization. Journal of Experimental Psychology: Human Perception \& Performance, 7, $1031-1058$.

Geffen, G., Mason, C., Butterworth, P., MClean, S., \& Clark, C. R. (1996). Tactile simultaneity within and between the hemispheres: The effects of hemispace. Cognitive Neuropsychology, 13, 257-276.

GoldBerG, E., \& CoSTA, L. D. (1981). Hemispheric differences in the acquisition and use of descriptive systems. Brain \& Language, 14, 144-173.

Goldman, P. S., Lodge, A., Hammer, L. R., Semmes, J., \& Mishkin, M. (1968). Critical flicker frequency after unilateral temporal lobectomy in man. Neuropsychologia, 6, 355-366.

Gregory, R. L., \& HARRIS, J. P. (1984). Real and apparent movement nulled. Nature, 307, 729-730.

Grondin, S. (1998), Judgments of the duration of visually marked empty time intervals: Linking perceived duration and sensitivity. Perception \& Psychophysics, 60, 319-330.

Gur, R. B., Packer, I. K., Hungerbuhler, J. P., Reivich, M., Obrist, W. D., Amarnek, W. S., \& SaCkeim, H. A. (1980). Differences in the distribution of gray and white matter in human cerebral hemispheres. Science, 207, 1226-1228.

Hammond, G. R. (1981). Finer temporal acuity for stimuli applied to the preferred hand. Neuropsychologia, 19, 325-329.

JASPER, H. H., \& RANEY, E. T. (1937). The phi test of lateral dominance. American Journal of Psychology, 49, 450-457.

KimuRA, D. (1966). Dual function asymmetry of the brain in visual perception. Neuropsychologia, 4, 275-285.

Kirman, J. H. (1974). Tactile apparent movement: The effect of interstimulus onset interval and stimulus duration. Perception \& Psychophysics, 15, 1-6.

LACKNER, J. R., \& TEUBER, H. L. (1973). Alterations in auditory fusion thresholds after cerebral injury in man. Neuropsychologia, 11, 409415.

Mason, C., \& GefFen, G. (1996). Temporal integration of events within and between the cerebral hemispheres. Cortex, 32, 97-108. 
MCNiCOL, D. (1972). A primer of signal detection analysis. Sydney: Australasian Publishing.

MILLER, R. (1996). Axonal conduction time and human cerebral laterality: A psychobiological theory. Amsterdam: Harwood.

Mills, L., \& Rollman, G. B. (1980). Hemispheric asymmetry for auditory perception of temporal order. Neuropsychologia, 18, 41-47.

Moscovitch, M. (1986). Afferent and efferent models of visual perceptual asymmetries: Theoretical and empirical implications. Neuropsychologia, 24, 91-114.

Nagarajan, S. S., Blake, D. T., Wright, B. A., Byle, N., \& Merzenich, M. M. (1998). Practice-related improvements in somatosensory interval discrimination are temporally specific but generalise across skin location, hemisphere and modality. Journal of Neuroscience, 18, 1559-1570.

NicholLs, M. E. R. (1994a). Hemispheric asymmetries for temporal resolution: A signal detection analysis of threshold and bias. Quarterly Journal of Experimental Psychology, 47A, 291-310.

NichOLLS, M. E. R. (1994b). The non-contribution of attentional biases to visual field asymmetries for temporal discrimination. Neuropsychologia, 32, 209-220.

NicholLs, M. E. R. (1996). Evidence and implications of a temporal processing dichotomy between the hemispheres. Laterality, 1, 97 . 137.

Nicholls, M. E. R., \& CoOper, C. J. (1991). Hemispheric differences in the rate of information processing for simple non-verbal stimuli. Neuropsychologia, 29, 677-684.

Nicholls, M. E. R., Schier, M., Stough, C. K. K., \& Box, A. (1999). Psychophysical and electrophysiological support for a left hemisphere temporal-processing advantage. Neuropsychiatry, Neuropsychology \& Behavioural Neurology, 12, 11-16.

Nicholls, M. E. R. \& Whelan, R. E. (1998). Hemispheric asymmetries for the temporal resolution of brief tactual stimuli. Journal of Clinical \& Experimental Neuropsychology, 20, 445-456.

Nicoletti, R., Anzola, G. P., Luppino, G., Rizzolatti, G., \&
UMILTÀ, C. (1982). Spatial compatibility effects on the same side of the body midline. Journal of Experimental Psychology: Human Perception \& Performance, 8, 664-673.

OLDFIELD, R.C. (1971). The assessment of handedness: The Edinburgh Inventory. Neuropsychologia, 9, 97-133.

Papcun, G., Krashen, S., Terbeek, D., Remmington, R., \& HarshMAN, R. (1974). Is the left hemisphere specialized for speech, language and/or something else? Journal of the Acoustical Society of America, 55, 319-327.

Parsons, O. A., Majumder, R. K., \& Chandler, P. J. (1967). Impaired flicker detection in visual fields subserved by non-damaged hemispheres. Cortex, 3, 307-316.

Reuter-Lorenz, P. A.. Kinsbourne, M., \& Moscovitch, M. (1990). Hemispheric control of spatial attention. Brain \& Cognition, 12, 240266.

SadLER, A. J., \& DeARY, I. J. (1996). Cerebral asymmetries in inspection time? Neuropsychologia, 34, 283-295.

ST. JoHN, R. (1998). Judgements of visual precedence by strabismics. Behavioural Brain Research, 90, 167-174.

SwISHER, L., \& HIRSH, I. J. (1972). Brain damage and the ordering of two temporally successive stimuli. Neuropsychologia, 10, 137-152.

Szelag, E., Steinbüchel, M., \& PöPPel, E. (1997). Temporal processing disorders in patients with Broca's aphasia. Neuroscience Letters, 235, 33-36.

TAYlor, M. M., \& CREelman, C. D. (1967). PEST: Efficient estimates on probability functions. Journal of the Acoustical Society of America, 41, 782-787.

Vroon, P. A., Timmers, H., \& Tempelaars, S. (1977). On the hemispheric representation of time. In S. Dornic (Ed.), Attention and performance VI (pp. 231-245). Hillsdale, NJ: Erlbaum.

(Manuscript received December 15, 1998; revision accepted for publication May 26, 1999.) 